\title{
Protocolo Clínico para Seleção de Candidatos ao Transplante de Ilhotas
}

\section{artigo original}

\author{
Luciana S. Percegona \\ Carlos A. M. AItA \\ Ester Pereira \\ ELAINE D. SOTTA \\ IRENICE C. DA SILVA \\ Miguel C. Riella
}

Laboratório de Isolamento de Ilhotas Humanas da Pontifícia

Universidade Católica do Paraná (PUC-PR), Curitiba, PR, Brasil (LSP, CAMA, EDS, ICS, MCR); Fundação Pró-Renal, Curitiba, PR, Brasil (LSP, EP, MCR).

\section{RESUMO}

Objetivo: Descrever o perfil clínico dos candidatos ao programa de transplante de ilhotas da Pontifícia Universidade Católica do Paraná (PUC-PR), em Curitiba. Métodos: $O$ processo de avaliação clínica foi estruturado em etapas: triagem, pré-avaliação, avaliação e lista de espera. Os critérios de inclusão utilizados foram: ocorrência de hipoglicemia assintomática, complicações crônicas progressivas da doença, idade entre 18 e 65 anos e pelo menos cinco anos de doença. Resultados: De setembro de 2003 a setembro de 2006 foram avaliados 92 candidatos, dos quais 25 preencheram os critérios de triagem, sendo selecionados para pré-avaliação. 0 principal motivo de não qualificação foi o uso de insulina em dose $>0,7 \mathrm{UI} / \mathrm{kg} / \mathrm{d}$. Dos 25 candidatos incluídos na pré-avaliação, sete não concordaram em assinar o termo de consentimento. Quatro candidatos completaram todas as etapas de seleção, porém apenas dois permanecem em lista de espera. Conclusões: Os candidatos ao transplante de ilhotas devem ser rigorosamente selecionados. Dois pacientes preencheram todos os critérios e encontram-se em lista de espera. (Arq Bras Endocrinol Metab 2008; 52/3:506-514)

Descritores: Diabetes melito do tipo 1; Transplante de ilhotas; llhotas de Langerhans; Triagem

\section{ABSTRACT}

Clinical Protocol for Selection of the Candidates for Islet Transplantation. Background: Our intention is to describe the clinical profile of the candidates for islet transplantation in Curitiba, Brazil. Methods: The clinical evaluation was organized in stages: Screening, Initial Evaluation, Evaluation and Waiting List. Candidates' inclusion criteria were hypoglycemia unawareness, glycemic imbalance, chronic progressive diabetic complications, 18-65 years of age and at least 5 years of type 1 diabetes evolution. Results: From September 2003 through September 2006, 92 candidates were clinically evaluated, and 25 fulfilled the Screening criteria, being selected at this stage. The main reason for exclusion was insulin requirement of more than $0.7 \mathrm{lU} / \mathrm{kg} /$ day. At the Initial Evaluation, seven of the 25 patients were excluded as have not agreed to sign the informed consent. Until now, 4 candidates completed the Evaluation stage and two of them are currently enlisted. Conclusions: Candidates for islet transplantation must be rigorously evaluated. Two patients fulfilled all the selection criteria and are currently enlisted. (Arq Bras Endocrinol Metab 2008; 52/3:506-514)

Recebido em 17/01/2007 Aceito em 21/09/2007
Keywords: Type 1 diabetes mellitus; Islet transplantation; Islets of Langerhans; Triage 


\section{INTRODUÇÃo}

DiABETES MELITO TIPO l (DMl) é uma doença que atinge aproximadamente $0,2 \%$ da população mundial e tem como principal característica a elevação dos níveis sanguíneos de glicose. Este aumento da glicemia decorre, basicamente, de uma deficiência sistêmica de insulina, conseqüente à destruição das células- $\beta$ das ilhotas pancreáticas $(1,2)$.

O tratamento convencional do DMl tem como base a reposição de insulina exógena, geralmente realizada por meio de injeções subcutâneas. Os protocolos mais modernos introduziram esquemas intensivos de reposição, seja por meio de múltiplas aplicações diárias ou por meio de bombas de insulina de infusão contínua subcutânea, com o objetivo de melhorar o controle glicêmico dos pacientes $(3,4)$. Mesmo assim, como esse tipo de terapia não consegue reproduzir fielmente o padrão fisiológico de secreção de insulina do pâncreas, os pacientes ficam expostos a riscos aumentados de desenvolvimento de complicações agudas, como a hipoglicemia, e de complicações crônicas, como nefro, retino e neuropatias (5). Entre as alternativas terapêuticas à insulinoterapia exógena disponíveis atualmente, somente o transplante de pâncreas (órgão total) e o de ilhotas pancreáticas possibilitam a reconstituição do padrão fisiológico de secreção de insulina (6). A partir do ano de 2000, com a introdução de uma nova técnica que ficou conhecida como protocolo de Edmonton, o transplante de ilhotas vem se firmando como uma alternativa eficaz para o tratamento de casos selecionados de DMl $(7,8)$. A principal vantagem do transplante de ilhotas consiste em envolver um procedimento cirúrgico pouco invasivo e bastante seguro, que praticamente não apresenta riscos, pois somente o tecido endócrino (que corresponde a aproximadamente $1 \%$ a $2 \%$ do pâncreas) é transplantado (9).

Com o objetivo de oferecer esta alternativa terapêutica aos pacientes diabéticos, foi criado na cidade de Curitiba o Laboratório de Isolamento de Ilhotas Humanas, no campi da Pontifícia Universidade Católica do Paraná (PUC-PR) (10). A construção e a aquisição dos equipamentos deste laboratório, bem como a contratação e o treinamento dos recursos humanos necessários, foi resultado de uma iniciativa conjunta da Secretaria de Estado de Ciência, Tecnologia e Ensino Superior do Paraná (SETI), da PUC-PR e da Fundação Pró-Renal. O laboratório iniciou suas atividades de isolamento e cultivo de ilhotas no ano de 2004 , tendo realizado o primeiro transplante em novembro de 2005 .
O objetivo deste trabalho é descrever os resultados obtidos até o momento com a seleção de pacientes e a implementação do programa clínico de transplante associado ao Laboratório de Isolamento de Ilhotas $\mathrm{Hu}^{-}$ manas da PUC-PR.

\section{MÉTODOS}

\section{População-alvo}

O projeto foi elaborado com a intenção de oferecer a terapia de transplante de ilhotas a pacientes com DMl apresentando hipoglicemias assintomáticas freqüentes, controle metabólico instável ou complicações crônicas progressivas da doença (apesar de controle glicêmico adequado), conforme descrito nos critérios de inclusão na Tabela 1 . O projeto propõe a realização do transplante em 12 pacientes com estas características. A população-alvo foi definida segundo o protocolo de Edmonton original, não contemplando neste momento, pacientes diabéticos com insuficiência renal. Todos os protocolos clínicos e laboratoriais descritos no projeto foram aprovados pelo Comitê de Ética em Pesquisa (CEP) do Centro de Ciências Biológicas e da Saúde da PUC-PR e pela Comissão Nacional de Ética em Pesquisa (Conep). O programa de seleção iniciou suas atividades em setembro de 2003 e para o presente trabalho foram considerados os dados analisados até setembro de 2006.

\section{Divulgação}

A divulgação do projeto foi realizada por meio de notícias em jornais, televisão e no site da Fundação PróRenal (http://www.pro-renal.org.br), sendo os candidatos orientados a entrar em contato telefônico com a Fundação ou com a Secretaria do Laboratório de Isolamento de Ilhotas Humanas da PUC-PR para informações adicionais.

\section{Questionário e material didático}

Aos candidatos que demonstraram interesse e contataram o serviço foi enviado formulário contendo as seguintes questões: a) idade; b) sexo; c) peso; d) altura; e) tempo desde o diagnóstico da doença; f) acompanhamento médico; g) necessidade diária de insulina; h) quantidade diária de aplicações; i) hemoglobina glicada; j) freqüência de episódios de hipoglicemia; k) controle 
glicêmico domiciliar; 1) principais preocupações pessoais com a evolução ou complicações do diabetes; $\mathrm{m}$ ) motivação para realização do transplante; n) preocupações com os riscos e as complicações do transplante. Foi solicitado aos candidatos para realizarem medidas de glicemia capilar com glicosímetro portátil durante dois dias seguidos (sete/oito medidas a cada dia) e os resultados anotados em espaço reservado para este fim no formulário, juntamente com a dose e o tipo de insulina utilizada em cada momento. Os candidatos foram orientados a responder ao questionário com auxílio do seu médico diabetologista e enviá-lo ao Laboratório de Isolamento de Ilhotas da PUC-PR, acompanhado de um relatório médico. Juntamente com o formulário foi enviado mate-

Tabela 1. Critérios clínicos de inclusão e exclusão de pacientes para transplante de ilhotas pancreáticas humanas.

\section{Critérios de inclusão}

Diabetes melito tipo 1 há mais de 5 anos.

Idade entre 18 e 65 anos.

Controle metabólico instável: dois ou mais episódios de hipoglicemia grave ( $<54 \mathrm{mg} / \mathrm{dl}$ ) que necessite de auxílio e/ou duas ou mais hospitalizações por cetoacidose diabética no período de um ano.

Hipoglicemias (<54 mg/dl) assintomáticas ou que necessitem de auxílio.

Controle glicêmico inadequado ( $\mathrm{HbAlc}>$ 8,0\%), mesmo em tratamento insulínico intensivo (três ou mais aplicações diárias ou uso de bomba de insulina).

Complicações progressivas do diabetes apesar de controle glicêmico adequado: retinopatia não-proliferativa, nefropatia (albuminúria $\geq 20$ mg/min, nos últimos 3 meses) ou neuropatia autonômica progressiva ou persistente.

\section{Critérios de exclusão}

Idade $<18$ ou $>65$ anos

Diagnóstico do diabetes $<5$ anos

Sem acompanhamento médico por $>6$ meses

IMC $>26$ ou peso $>75 \mathrm{~kg}$ (homens) e $>70 \mathrm{~kg}$ (mulheres)

$\mathrm{HbAlc}>12 \%$

Necessidade insulínica $>0,7 \mathrm{UI} / \mathrm{kg} /$ dia

Peptídeo-C basal ou após estímulo $>0,3 \mathrm{ng} / \mathrm{ml}$

Clearance de creatinina $<60 \mathrm{ml} / \mathrm{min}$, creatinina sérica

$>1,6 \mathrm{mg} / \mathrm{dl}$ e/ou macroalbuminúria (> $300 \mathrm{mg} / 24 \mathrm{~h}$ )

Hemoglobina < 12 mg/dl, alteração na linhagem

Leucocitária ou plaquetária ou alteração coagulograma

Alteração da função hepática > 1,5 × valor normal

Colesterol LDL > $130 \mathrm{mg} / \mathrm{dl}$ ou triglicerídeos > $200 \mathrm{mg} / \mathrm{dl}$

Sorologia positiva para HIV, HTLV-1/2, hepatite B ou C

Vírus Epstein-Barr (EBV) positivo ou evidência de

Infecção aguda (lgM > lgG)

Toxoplasmose aguda (lgM > lgG)

Sorologia positiva para doença de Chagas

Qualquer doença infecciosa ativa

PPD positivo
Painel de alorreatividade (PRA) $>20 \%$

$\mathrm{PSA}>4 \mathrm{ng} / \mathrm{ml}$

Alteração da função tiroidiana

Radiografia de tórax anormal

Ecografia abdominal e pélvica anormal

Mamografia anormal

Instabilidade cardiovascular

Alteração de ecocardiograma de estresse em

pacientes $>35$ anos

Retinopatia diabética proliferativa

Transplante de órgão prévio

Doença maligna prévia

Exame odontológico anormal

Gravidez ou amamentação

Uso de droga ilícita

Tabagismo e/ou etilismo nos últimos 6 meses

Qualquer condição médica que necessite o

uso de esteróides

Qualquer situação (incluindo avaliação

psicológica) que contra-indique o transplante 
rial didático contendo informações sobre o transplante de ilhotas, sobre as medicações utilizadas, sobre os critérios de inclusão e exclusão de candidatos e sobre o consentimento para participação no projeto de pesquisa.

\section{Triagem}

Os questionários que retornaram foram analisados individualmente e utilizados para triagem inicial dos pacientes. Aqueles que preencheram os critérios (Tabela 1) de inclusão e não se enquadraram nos critérios de exclusão foram selecionados para a etapa de pré-avaliação. $\mathrm{O}$ índice de massa corpórea (IMC) foi calculado segundo a fórmula $\mathrm{IMC}=$ peso $(\mathrm{kg}) /$ altura $\left(\mathrm{m}^{2}\right)$. As medidas de glicemia capilar realizadas pelos candidatos foram utilizadas para o cálculo do MAGE (mean amplitude of glycemic excursions), conforme descrito por Service e cols. (11).

\section{Pré-avaliação}

Os candidatos selecionados na triagem inicial foram convidados a realizar entrevista e avaliação com a equi- pe médica. $\mathrm{Na}$ entrevista receberam informações adicionais sobre o projeto e o termo de consentimento livre e esclarecido (TCLE) para participação na pesquisa. A avaliação constou de anamnese, exame físico e solicitação de alguns exames complementares (Tabela 2a). Os candidatos foram também encaminhados para avaliação psicológica, que foi realizada pelo psicólogo da Fundação Pró-Renal.

\section{Avaliação}

$\mathrm{Na}$ consulta médica de retorno, os pacientes que preencheram os critérios clínicos e laboratoriais da etapa anterior foram selecionados e encaminhados para realização de novos exames complementares (Tabela 2b). Para estes pacientes foram também solicitados relatórios médicos atuais (do último ano) de avaliação odontológica, oftalmológica e ginecológica, esta última incluindo $\mathrm{Pa}$ panicolau e cultura endocervical. Para mulheres com idade superior a 40 anos ou história familiar de câncer de mama também foi solicitada mamografia.

Tabela 2. Exames complementares e escala de atualização dos parâmetros clínicos e laboratoriais realizados nas etapas de pré-avaliação, avaliação e lista de espera, para candidatos ao transplante de ilhotas.

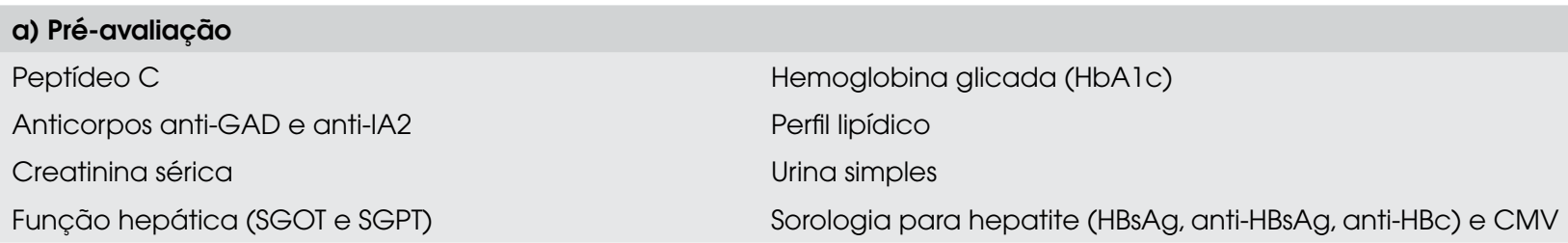

\section{b) Avaliação}

Sorologia para HIV
Eletrocardiograma
Radiografia de tórax
Urina 24 horas e clearance de creatinina

PRA

PPD

Ecocardiograma de estresse

Teste de estímulo de secreção de peptídeo C com Sustagem Endoscopia digestiva alta (se história prévia de úlcera gástrica)

\section{C) Lista de espera}

Mensalmente: PRA

Trimestralmente: Hemoglobina glicada ( $\mathrm{HbAlc})$

Semestralmente: Perfil lipídico sérico, função hepática e renal, urina 24 horas

Anualmente: História e exame físico, eletrocardiograma, sorologias para HIV, HBV, HCV, CMV, radiografia de tórax, PPD, ecografia abdominal, ecocardiograma de estresse (se idade acima de 35 anos), endoscopia digestiva alta (se história prévia de úlcera), avaliação oftalmológica, ginecológica e odontológica, mamografia (mulher com idade > 40 anos ou história familiar de câncer de mama) 


\section{Seleção para lista de espera}

Os pacientes aprovados em todos os critérios das etapas anteriores foram selecionados para inclusão em lista de espera para o transplante de ilhotas. Enquanto aguardavam em lista pelo transplante, aos pacientes foi solicitado realizar glicemias capilares diárias e enviar os resultados à equipe médica, juntamente com o registro da dose de insulina utilizada. Também houve indicação, aos pacientes, para cumprirem uma escala de atualização dos parâmetros clínicos e laboratoriais (Tabela 2c) no período de espera em lista.

\section{RESULTADOS}

\section{Questionário}

De todos os pacientes que entraram em contato para obter informações adicionais sobre o projeto, 369 soli- citaram o envio do formulário, porquanto 92 foram respondidos e enviados à equipe médica para análise.

\section{Triagem}

O resumo dos dados obtidos dos 92 candidatos que retornaram os questionários está apresentado nas Tabelas 3 e 4. Dos 92 candidatos, 25 preencheram os critérios da triagem. Estes candidatos foram selecionados e convidados por carta para participar da etapa de pré-avaliação. Os candidatos não qualificados receberam correspondência informando a sua não-inclusão. Por necessitarem $>0,7$ UI de insulina $/ \mathrm{kg} /$ dia não qualificaram para o transplante 18 candidatos (visto que 14 destes realizavam esta dose em uma ou duas aplicações diárias), 16 não apresentavam hipoglicemia assintomática, oito candidatos apresentavam IMC $>26$ ou peso inadequado, 10 eram menores de 18 anos ou acima de 65 anos, 10 apresentavam diabetes tipo 2 (DM2), três não estavam sob acompanhamento médico, três tinham menos de cinco anos de evolução do diabetes

Tabela 3. Perfil dos pacientes diabéticos candidatos a transplante de ilhotas (dados obtidos dos formulários enviados para análise).

\begin{tabular}{|c|c|}
\hline & Média \pm DP (mín. - máx.) \\
\hline Sexo & $M=48 F=44$ \\
\hline Idade (anos) & $33,8 \pm 15,1(5-71)$ \\
\hline Índice de massa corporal (IMC) & $23 \pm 4,4(15-41)$ \\
\hline Tempo diagnóstico do diabetes (anos) ${ }^{a}$ & $13,8 \pm 9,2(1-48)$ \\
\hline Necessidade diária de insulina (UI/kg/d) & $0,74 \pm 0,32(0,1-1,6)$ \\
\hline №̣ de aplicações diárias de insulinab & $\begin{array}{l}2,3 \pm 1,0 \\
1 \text { aplicação: } 14 \text { pacientes } \\
2 \text { aplicações: } 46 \text { pacientes } \\
3 \text { ou mais aplicações: } 27 \text { pacientes }\end{array}$ \\
\hline Hemoglobina glicada - HbAlc (\%) & $9,6 \pm 2,4(5-15)$ \\
\hline MAGE & $181,5 \pm 96,0(19,0-393,0)$ \\
\hline Automonitoração glicêmica (determinações/dia) ${ }^{c}$ & $2,22 \pm 0,9(0-5)$ \\
\hline Controle médico & $\begin{array}{l}\text { Sem médico = } 3(3,3 \%) \\
\text { Clínico-geral }=22(23,8 \%) \\
\text { Nefrologista }=2(2,2 \%) \\
\text { Endocrinologista }=60(65,2 \%) \\
\text { Cardiologista: } 2(2,2 \%) \\
\text { Pediatra = } 1(1,1 \%) \\
\text { Sem resposta }=2(2,2 \%)\end{array}$ \\
\hline
\end{tabular}


Tabela 4. Motivação e preocupação em relação ao transplante de ilhotas (dados obtidos dos formulários enviados para análise).

\begin{tabular}{|c|c|}
\hline & Número de pacientes \\
\hline $\begin{array}{l}\text { Principal preocupação em relação à evolução do } \\
\text { diabetes }\end{array}$ & $\begin{array}{l}\text { Complicações crônicas = } 82 \text { pacientes } \\
\text { Hipoglicemia = } 8 \text { pacientes } \\
\text { Coma = } 2 \text { pacientes }\end{array}$ \\
\hline Principal motivação para realização do transplante & $\begin{array}{l}\text { Qualidade de vida }=39 \text { pacientes } \\
\text { Insulino-independência = } 27 \text { pacientes } \\
\text { Dieta sem restrições = } 20 \text { pacientes } \\
\text { Medo da hipoglicemia = } 6 \text { pacientes }\end{array}$ \\
\hline Principais preocupações em relação ao transplante & $\begin{array}{l}\text { Rejeição = } 39 \text { pacientes } \\
\text { Efeitos colaterais das drogas }=14 \text { pacientes } \\
\text { Nenhuma preocupação }=21 \text { pacientes } \\
\text { Falta de doador: } 1 \text { paciente } \\
\text { Outras: } 17 \text { pacientes }\end{array}$ \\
\hline
\end{tabular}

e um apresentava controle glicêmico inadequado. Destacamos que alguns candidatos apresentaram mais de um motivo para não qualificação. Os pacientes não qualificados foram orientados a procurar auxílio ou retornar ao médico de origem para o acompanhamento clínico adequado.

\section{Pré-avaliação}

Dos 25 pacientes incluídos nesta etapa, sete foram selecionados e convidados para participar da etapa de avaliação. Dos 18 candidatos não qualificados, sete não concordaram em assinar o TCLE, três foram reprovados na avaliação psicológica, um apresentava insuficiência renal, quatro não apresentavam hipoglicemia assintomáti$\mathrm{ca}$, dois foram submetidos a transplante pancreático e um não quis participar do processo de seleção.

\section{Avaliação}

Dos sete pacientes incluídos nesta etapa, quatro completaram a avaliação. Todos os quatro apresentavam peptídeo $\mathrm{C}$ sérico $<0,5 \mathrm{ng} / \mathrm{ml}$ no teste de estímulo com Sustagen, MAGEs elevados $(337 \mathrm{mg} / \mathrm{dl}, 320 \mathrm{mg} / \mathrm{dl}$, $225 \mathrm{mg} / \mathrm{dl} \mathrm{e} 184 \mathrm{mg} / \mathrm{dl}$ ), hemoglobinas glicadas também elevadas $(9,5 \%, 8,2 \%, 11,6 \%$ e $9,4 \%)$ e episódios freqüentes de hipoglicemias assintomáticas. Dos candidatos não selecionados, um foi reprovado por apresentar hemangioma hepático, um apresentava sorologia positiva para HIV e um foi a óbito por morte súbita.

\section{Lista de espera}

Dos quatro pacientes selecionados inicialmente, apenas dois encontram-se em lista de espera para o transplante.
Um dos candidatos foi desqualificado por instabilidade e progressão da retinopatia diabética e outro por seguimento clínico irregular. Destes pacientes inicialmente listados, quatro (100\%) apresentavam episódios recorrentes de hipoglicemias graves, três (75\%) apresentavam instabilidade metabólica e dois (50\%) com complicações progressivas secundárias do DMl. A taxa de elegibilidade para o transplante, no nosso programa, foi de $4 \%$ (quatro pacientes, em 92 avaliados).

Observação: Dos pacientes listados até o momento, um foi submetido à primeira infusão de ilhotas, em novembro de 2005. No seguimento inicial (três meses) pós-transplante, o paciente mostrou redução em torno de $30 \%$ da dose diária de insulina.

\section{DISCUSSÃo}

Os transplantes de pâncreas e de ilhotas pancreáticas são as únicas alternativas terapêuticas disponíveis atualmente capazes de recompor o padrão fisiológico de secreção de insulina em pacientes diabéticos (6). Esse dado torna-se ainda mais relevante pelo reconhecimento de que a manutenção de níveis glicêmicos próximos da normalidade é de extrema importância para evitar o desenvolvimento ou mesmo reverter, das complicações crônicas decorrentes da doença (5).

A partir do ano de 2000 , com a introdução do protocolo de Edmonton, o transplante de ilhotas vem sendo considerado uma alternativa terapêutica eficaz para o tratamento do DMl (7). Esse protocolo, desenvolvido por pesquisadores da Universidade de $\mathrm{Al}$ 
berta, em Edmonton, no Canadá, introduziu um novo esquema de drogas imunossupressoras, que não utiliza corticosteróides (que são drogas diabetogênicas), associado à infusão intra-hepática de grande massa de ilhotas. Para obter a quantidade adequada de células, geralmente é necessário o processamento de dois a três pâncreas de doadores, e as ilhotas isoladas de cada órgão são transplantadas em infusões independentes. A infusão é realizada na veia-porta, por meio de punção percutânea trans-hepática, em que as ilhotas acabam impactando em pequenos capilares portais, ocorre neovascularização e no fígado passam a produzir e secretar insulina.

A taxa de sucesso na descrição inicial deste protocolo, medida pela insulino-independência no seguimento de um ano após o transplante, alcançava os $100 \%$. Porém, os relatórios mais recentes de grupos norte-americanos e canadenses que se formaram com o objetivo de reproduzir os resultados iniciais são menos otimistas, de aproximadamente $58 \%$ de sucesso no primeiro ano. Esses dados estão disponíveis no site do Collaborative Islet Transplant Registry (CITR) (http:// spitfire.emmes.com/study/isl/reports/reports.htm). Apesar de menor porcentagem dos pacientes avaliados neste estudo atingir insulino-independência, todos apresentaram produção de peptídeo $\mathrm{C}$ no seguimento pós-transplante, e também melhora nos valores de hemoglobina glicada e redução drástica nos episódios de hipoglicemia, reforçando a validade dessa técnica na melhora do controle glicêmico e da qualidade de vida de pacientes diabéticos.

Os resultados obtidos pelo grupo de Edmonton levaram à formação de diversos outros centros de transplante de ilhotas no mundo inteiro. Estima-se que existam, atualmente, mais de 60 grupos, sendo a metade deles nos Estados Unidos, e mais de 300 procedimentos realizados após o ano de 2000 , fundamentados no protocolo de Edmonton ou em modificações deste.

Com o objetivo de oferecer essa modalidade de tratamento na cidade de Curitiba, foi inaugurado no ano de 2002 o Laboratório de Isolamento de Ilhotas Humanas, nos campi da PUC-PR. A construção e a aquisição de equipamentos para o laboratório, bem como o treinamento da equipe necessária para o desenvolvimento do projeto, foi resultado de uma cooperação entre a Secretaria de Estado de Ciência, Tecnologia e Ensino Superior do Paraná (SETI), da PUC-PR e da Fundação Pró-Renal (10). O treinamento médico para a seleção e o acompanhamento pré e pós-transplante de pacientes foi realizado no Diabetes Research Institute, em Miami, um dos principais centros de referência em transplante de ilhotas.

Pelo fato de as indicações clínicas atuais de consenso para transplante de ilhotas serem bastante específicas e restritas, a seleção de pacientes candidatos é uma das etapas mais importantes no desenvolvimento de um programa clínico de transplante.

A proposta deste projeto é realizar o transplante isolado de ilhotas em 12 pacientes diabéticos afetados por episódios freqüentes de hipoglicemias assintomáticas, instabilidade metabólica ou complicações crônicas progressivas, com base no protocolo original desenvolvido em Edmonton. Pelo fato de não contemplar pacientes diabéticos com insuficiência renal candidatos a transplantes combinados de rim e ilhotas, a seleção correta de candidatos torna-se ainda mais importante. Para que o transplante isolado seja vantajoso ao receptor, os seus potenciais benefícios, como a regularização do controle glicêmico (tendo a insulinoindependência como meta), a redução dos episódios de hipoglicemia e a estabilização ou a reversão das complicações crônicas, devem superar os potenciais efeitos adversos da imunossupressão (aumento da suscetibilidade a infecções e ao desenvolvimento de câncer, entre outros).

A divulgação inicial do projeto tem sido realizada em vários meios de comunicação, como jornais e televisão, sempre destacando prioritariamente a populaçãoalvo a que se destina. Depois do primeiro contato com o programa, é encaminhado um questionário aos candidatos que mostram interesse, além do convite para assistir a uma apresentação sobre o projeto de transplante de ilhotas.

De uma grande quantidade de contatos (principalmente telefônicos e pessoais) ao serviço para obtenção de informações adicionais, 369 candidatos até o momento solicitaram o envio do formulário e 92 os devolveram respondidos.

Entre as principais motivações descritas pelos candidatos para realização do transplante, destacam-se: a melhora da qualidade de vida, a obtenção de insulinoindependência e a dieta sem restrições. Quanto às principais preocupações dos candidatos em relação a esta terapia, temos: o risco de rejeição e os efeitos colaterais das drogas. Essas informações fazem supor que as restrições impostas pela doença promovem desconforto suficiente, a ponto de relegar, a segundo plano, as potenciais complicações secundárias ao transplante. 
O principal motivo de não qualificação para o transplante entre esses candidatos foi o uso de insulina em doses diárias $>0,7 \mathrm{UI} / \mathrm{kg}$, o que foi observado em 18 pacientes. Desses pacientes, 13 realizavam a dose diária dividida em uma ou duas aplicações, o que certamente deve ter contribuído para a ocorrência dos episódios freqüentes de hipoglicemia descritos pelos pacientes. Um dado importante é que três dentre 14 pacientes não se encontravam sob acompanhamento médico e os 11 demais, apesar de relatarem acompanhamento por endocrinologista ou clínico-geral, no momento não estavam seguindo as condições ideais de tratamento para DMl preconizadas pela Associação Americana de Diabetes e por grande parte dos diabetologistas, que propõe múltiplas ( 3 a 4 ) aplicações diárias (4). Apenas 27 pacientes $(30 \%)$ encontravam-se em esquema de tratamento intensivo. Do mesmo modo, os valores médios de hemoglobina glicada e de MAGE observados no grupo geral de candidatos $(10,0 \%$ e $181,5 \mathrm{mg} / \mathrm{dl}$, respectivamente) também sugerem controle inadequado da doença. Os pacientes não qualificados eram orientados a procurar o médico diabetologista para receber acompanhamento clínico adequado. Apenas para reforçar a informação descrita anteriormente, nenhum dos 92 candidatos analisados se encontrava sob tratamento com bomba de insulina, que é considerado um dos métodos atuais mais efetivos para manutenção de controle glicêmico ideal (3).

Apesar de não ser possível avaliar a representatividade deste grupo em relação à população brasileira ou mesmo local de DMl, os dados sugerem que, em geral, o acompanhamento desses pacientes não é adequado. É possível também supor a necessidade urgente de políticas públicas eficientes que revertam esse quadro e, assim, reduzam a incidência de complicações crônicas da doença e seu custo elevado sobre os sistemas público e privado de saúde. Aqui é importante também ressaltar que a principal preocupação descrita pelos candidatos ao transplante (quase a totalidade), em relação à evolução do diabetes, foi o desenvolvimento de complicações crônicas da doença.

Até o momento, quatro pacientes completaram toda a avaliação e foram listados para realização do transplante de ilhotas. Entretanto, no seguimento mensal foi observado em um dos pacientes a progressão e a instabilidade da retinopatia. Esse paciente permanece atualmente como inativo. Em outro caso, o paciente foi desqualificado por não aderir ao seguimento clínico solicitado. Dos pacientes inicialmente listados, quatro
(100\%) apresentavam hipoglicemias graves, três $(75 \%)$ instabilidade metabólica e dois (50\%) complicações progressivas secundárias do DMl.

No nosso programa, até o momento, um dos pacientes listados foi submetido ao transplante de ilhotas. No seguimento inicial de três meses pós-transplante, este paciente apresentou redução em torno de $30 \%$ na necessidade diária de insulina. Também mostrou melhora no controle glicêmico, com redução da taxa de hemoglobina glicada para 7\% e ausência de episódios de hipoglicemia assintomática. A continuidade do nosso programa nessa fase de transplante clínico permitirá melhor avaliação da nossa estratégia de seleção de candidatos.

Na literatura médica, apenas uma publicação recente foi encontrada com enfoque específico na seleção de candidatos para transplante de ilhotas, o que torna um pouco difícil a comparação dos nossos resultados com os de outros centros. Nesse trabalho, realizado por um grupo francês, os autores avaliaram 79 candidatos (sendo 41 para transplante isolado) em um período de cinco anos (12). Dos 41 candidatos a transplante isolado, 20 procuraram voluntariamente o serviço, oito foram contatados durante hospitalização por descontrole glicêmico e 13 foram indicados pelo diabetologista responsável. Para 14 dos 41 candidatos (34\%), o transplante não foi indicado por apresentarem controle glicêmico adequado com insulina injetável, peptídeo C sérico detectável, problemas renais ou hepáticos, ou intenção de engravidar. Dos 41 candidatos (39\%), 16 não se interessaram pelo transplante, sete mostraram mais interesse pela bomba de insulina. Dos 41 candidatos (27\%), 11 preencheram todos os critérios de inclusão, porquanto oito continuavam em avaliação, um estava em lista de espera e dois foram transplantados. Considerando todos os candidatos avaliados no estudo, 25\% se enquadraram nos critérios de inclusão e prosseguiram a avaliação, e $12 \%$ (10 pacientes, sendo oito transplantes combinados de rim e ilhotas e dois somente de ilhotas) foram transplantados.

Shapiro e cols., em 2006, divulgaram os resultados do International Trial of the Edmonton Protocol for Islet Transplantation, um estudo multicêntrico de transplante de ilhotas, envolvendo nove grupos dos Estados Unidos, do Canadá e da Europa (13). Nesse estudo foram avaliados 2 mil candidatos ao transplante, visto que apenas $149(7 \%)$ preencheram os critérios de inclusão. Desses 149 pacientes, 36 foram transplantados, tendo como indicação hipoglicemia grave em 35 pacientes (97\%), instabilidade glicêmica em 20 casos 
(56\%) e progressão das complicações secundárias do diabetes em outros 19 (53\%). Apesar de as diferenças entre as populações avaliadas, a taxa de elegibilidade no nosso programa, de $4 \%$, foi bastante próxima à obtida nesse estudo multicêntrico.

As principais indicações atuais para o transplante de ilhotas isolado são a ocorrência de hipoglicemias assintomáticas recorrentes e a labilidade do controle glicêmico. Como a quantificação destes fatores é bastante subjetiva, os pesquisadores do grupo de Edmonton descreveram, em 2004, um método de avaliação fundamentado em escores de hipoglicemia (freqüência, gravidade, intensidade das hipoglicemias assintomáticas) e labilidade glicêmica (variação nos níveis glicêmicos em relação à avaliação clínica), comparando os pacientes que foram submetidos ao transplante de ilhotas com um grupo-controle de pacientes de sua clínica-geral de DMl (14). Por causa dos riscos associados à imunossupressão, é importante que a seleção dos candidatos seja realizada de maneira rigorosa, garantindo que sejam submetidos ao procedimento somente aqueles pacientes com maior probabilidade de se beneficiarem com o transplante.

A importância deste trabalho foi desenvolver um critério objetivo para avaliação da hipoglicemia e instabilidade metabólica, que poderá permitir a indicação clínica mais precisa do transplante e comparações entre a seleção de candidatos de diversos centros. A implementação deste método pode ficar como uma perspectiva para a continuação do trabalho no nosso serviço e, desse modo, melhorar a assistência prestada aos nossos pacientes.

Finalizando, esperamos que os resultados deste trabalho possam ser úteis para a consolidação do programa clínico de transplante de ilhotas desenvolvido na cidade de Curitiba, bem como para os demais grupos que eventualmente venham a ter esta intenção.

\section{AGRADECIMENTOS}

Nossos sinceros agradecimentos à Sra . Rosângela Aparecida da Silva pela eficiente assistência administrativa e especial atenção no contato com os pacientes.

\section{REFERÊNCIAS}

1. Atkinson M, Eisenbarth G. Type 1 diabetes: new perspectives on disease pathogenesis and treatment. Lancet. 2001;358:221-9.

2. Devendra D, Liu E, Eisenbarth GS. Type 1 diabetes: recent developments. BMJ. 2004;328(7442):750-4.

3. American Diabetes Association: clinical practice recommendations. Continuous subcutaneous insulin infusion. Diabetes Care. 2004;27 Suppl 1:S110.

4. American Diabetes Association: clinical practice recommendations. Insulin administration. Diabetes Care. 2004;27 Suppl 1:S106-9.

5. The diabetes control and complications trial research group. The effect of intensive treatment of diabetes on the development and progression of long-term complications in insulin-dependent diabetes mellitus. N Engl J Med. 1993;329(14):977-86.

6. Bottino R, Trucco M, Balamurugan AN, Starzl TE. Pancreas and islet cell transplantation. Best Pract Res Clin Gastroenterol. 2002;16(3):457-74.

7. Shapiro AM, Lakey JR, Ryan EA, Korbutt GS, Toth E, Warnock $\mathrm{GL}$, et. al. Islet transplantation in seven patients with type 1 diabetes mellitus using a glucocorticoid-free immunosuppressive regimen. N Engl J Med. 2000;343(4):230-8.

8. Ryan EA, Lakey JR, Paty BW, Imes S, Korbutt GS, Kneteman NM, et. al. Successful islet transplantation: continued insulin reserve provides long-term glycemic control. Diabetes. 2002;51(7):2148-57.

9. Shapiro AM, Ryan EA, Lakey JR. Diabetes. Islet cell transplantation. Lancet. 2001;358 Suppl:S21.

10. Sotta ED, Madalozzo TM, Percegona L, Pereira J, Bignelli A, Senegaglia $A$, et. al. Establishing an islet transplantation program in a developing country. Transplant Proc. 2004;36(6):1700-03.

11. Service FJ, O'Brien PC. The relation of glycaemia to the risk of development and progression of retinopathy in the Diabetic Control and Complications Trial. Diabetologia. 2001;44(10):1215-20.

12. Vantyghem MC, Hazzan M, Tourvieille $S$, Provost F, Perimenis P, Declerck N, et. al. Selection of diabetic patients for islet transplantation. A single-center experience. Diabetes Metab. 2004;30(5):417-23.

13. Shapiro AMJ, Ricordi C, Hering BJ, Auchincloss $H$, Lindblad R, Robertson $P$, et. al. International Trial of the Edmonton Protocol for Islet Transplantation. N Engl J Med. 2006;355(13):1318-30.

14. Ryan EA, Shandro T, Green K, Paty BW, Senior PA, Bigam D, et. al. Assessment of the severity of hypoglycemia and glycemic lability in type 1 diabetic subjects undergoing islet transplantation. Diabetes. 2004;53(4):955-62.

Endereço para correspondência:

Luciana Soares Percegona

Rua Nunes Machado, 472, sala 1003

80.250-000 - Curitiba PR

E-mail: I.percegona@pucpr.br 